\title{
Recurrencia/persistencia de neoplasia intraepitelial cervical posterior a cono LEEP en el Instituto Nacional de Enfermedades Neoplásicas
}

\author{
Gino Venegas-Rodríguez ${ }^{1, a}$, Kennlly Cardoza-Jiménez ${ }^{2, b}$, Manuel Alvarez $^{3, a}$, Carlos Santos ${ }^{3, a}$, Julio C. Mariátegui ${ }^{3, a}$, \\ Carlos Velarde ${ }^{3, a}$, Henry Valdivia, ${ }^{3, a}$, Vicente A Benites-Zapata ${ }^{4, c}$
}

\section{RESUMEN}

Objetivo: Determinar la incidencia de recurrencia/persistencia de neoplasia intraepitelial cervical (NIC) en pacientes sometidos a procedimiento de escisión electroquirúrgica en asa (cono LEEP) en el Servicio de Ginecología Oncológica del Instituto Nacional de Enfermedades Neoplásicas (INEN).

Materiales y métodos: Estudio de cohorte, retrospectivo en el cual se revisaron las historias clínicas de pacientes sometidos a cono LEEP durante el periodo de enero del 2002 a diciembre del 2003. Los pacientes fueron divididos en un grupo con margen comprometido y otro con margen negativo después del procedimiento de cono LEEP. El seguimiento de los pacientes se realizó cada 3 meses con examen físico, PAP y colposcopía. El desenlace fue la recurrencia de NIC. Para valorar el riesgo de recurrencia de NIC entre los grupos de estudio se calculó el riesgo relativo (RR) con su respectivo intervalo de confianza (IC) al $95 \%$.

Resultados: En total 451 pacientes fueron sometidos a cono LEEP durante el periodo de estudio. La edad promedio fue 35.32 años, el promedio de parejas sexuales fue 2.1 y el promedio de hijos fue 2.7. Presentaron margen comprometido $109(25 \%)$ y margen negativo 342 (75\%). De ellos solo 94 y 102, respectivamente, cumplían los criterios de elegibilidad y fueron seguidos. La media de seguimiento fue de 21.74 meses. La incidencia de recurrencia/persistencia dentro de los pacientes con margen comprometido fue $5.5 \%$ mientras que la incidencia de recurrencia en un grupo de pacientes con margen negativo fue $1.96 \%$ No encontramos diferencias significativas en el riesgo de padecer recurrencia de NIC en el grupo de pacientes con margen comprometido versus los pacientes con margen negativo. RR=2.7; IC 95\% (0.41 a 33.8). Conclusión: No se encontró diferencia significativa en la incidencia de neoplasia intracervical para pacientes con margen comprometido y los pacientes con margen negativo.

Palabras clave: Neoplasia intraepitelial cervical; recurrencia; cono LEEP.

\section{Recurrence/persistence of cervical intraepithelial neoplasia after undergoing a LEEP conization at Instituto Nacional de Enfermedades Neoplásicas}

\author{
ABSTRACT
}

Objective: To determine the incidence of recurrence/persistence of cervical intraepithelial neoplasia (CIN) in patients who underwent a loop electrosurgical excision procedure (LEEP) at the Department of Gynecologic Oncology of Instituto Nacional de Enfermedades Neoplásicas (INEN).

Materials and methods: A retrospective cohort study in which medical records of patients who underwent a LEEP conization from January 2002 to December 2003 were examined. After the LEEP conization, patients were divided into two groups: one with positive margin and another one with negative margin. Follow-up was performed every 3 months and consisted of a physical examination, Pap test and colposcopy. The outcome was recurrence of CIN. To assess the risk of CIN recurrence among the study groups, relative risk (RR) and its respective 95\% confidence interval $(\mathrm{Cl})$ were calculated. Results: A total of 451 patients underwent a LEEP conization during the study period. The mean age was 35.32 years old, the mean number of sexual partners was 2.1 and the mean number of children was 2.7. One hundred and nine (109) patients (25\%) showed a positive margin and 342 (75\%) showed a negative one. Out of these subjects, only 94 and 102 , respectively, met the eligibility criteria and were followed. The mean follow-up period was 21.74 months. The incidence of recurrence/persistence in patients with positive margin was $5.5 \%$, while the incidence of recurrence/persistence in the group of patients with negative margin was $1.96 \%$. We found no significant differences in the risk of CIN recurrence in the group of patients with positive margin versus patients with negative margin. $\mathrm{RR}=2.7,95 \% \mathrm{Cl}(0.41$ to 33.8$)$. Conclusion: There was no significant difference in the incidence of cervical intraepithelial neoplasia between patients with positive margin and patients with negative margin.

Keywords: Cervical intraepithelial neoplasia; recurrence; LEEP conization.

1. Liga Peruana de Lucha contra el Cáncer. Lima, Perú.

2. Sociedad Científica de Estudiantes de Medicina, Universidad Nacional de Piura. Piura, Perú.

3. Instituto Nacional de Enfermedades Neoplásicas.

4. Centro de Investigación de Salud Pública, Instituto de Investigación, Facultad de Medicina Humana, Universidad de San Martín de Porres. Lima, Perú.

a. Ginecólogo Oncólogo.

b. Médico Cirujano.

c. Médico Epidemiólogo. 


\section{INTRODUCCIÓN}

El cáncer de cuello uterino es un problema de salud pública especialmente en países de bajos recursos, se estima que 7 mujeres mueren en el Perú cada día por esta causa. El cáncer de cuello uterino es precedido por un conjunto de lesiones cervicales llamadas Neoplasia Intraepitelial Cervical (NIC), las cuales se presentan por encima de la membrana basal del epitelio cervical y se dividen en NIC 1,2 y 3 en funcion del compromiso del tejido desde la membrana basal hacia arriba, determinando que para NIC1 el compromiso desde la membrana basal hasta el tercio inferior, NIC 2 compromiso desde la membrana basal hasta el tercio medio y NIC3 desde la membrana basal hasta la superficie; cuando el compromiso tisular atravieza la membrana basal determina la enfermedad infiltrante cervical $^{(1)}$.

La incidencia de las NIC en pacientes sometidas a tamizaje con citología varía entre 1,1 a $3,7 \%^{(1)}$ y el tratamiento de estas implica la destrucción o escisión de la zona de transformación anormal.

La citología combinada con los test virales moleculares para la identificación de la infección del Papiloma virus ha resultado en un importante incremento en los casos de NIC diagnosticados en las últimas décadas ${ }^{(1)}$.

Las opciones de tratamiento incluyen conización fría, conización láser, cono LEEP (loop eletrosurgical escision proceduce) o LLETZ (large loop excision of the transformation zone), crioterapia, cono láser y en algunos casos histerectomía ${ }^{(2)}$.

Antiguamente el cono frío era el procedimiento estándar como procedimiento diagnóstico y de tratamiento ${ }^{(2)}$. En 1989 , Prendiville, adaptó un instrumento electro quirúrgico para remover la zona de transformación, este instrumento llamado LEEP/LLETZ, permite el tratamiento de las NIC, con buena tolerabilidad y eficacia y que comparado con el cono frío es mucho menos costoso ${ }^{(3)}$. A pesar de la efectividad del LEEP/ LLETZ, la recurrencia de NIC varía del 5 al $64 \%$ de los pacientes ${ }^{(4,5)}$. El 74\% de los pacientes con NIC 2-3 tratados, presentan recurrencia en los primeros 6 meses ${ }^{(6,7)}$ Múltiples estudios reportan persistencia o recurrencia de NIC en mujeres con márgenes comprometidos por la enfermedad ${ }^{(8-10)}$. La habilidad para interpretar los márgenes de escisión es un componente crítico para determinar el manejo óptimo de pacientes tratados por esta patología y se ha mostrado que aproximadamente 10 al $20 \%$ de los especímenes obtenidos por cono LEEP/LLETZ son totalmente imposibles de interpretar, aparentemente consecuencia del daño térmico causado.

Si bien las indicaciones de cono LEEP/LLETZ están claras y su empleo es altamente aceptado, no existe consenso acerca del manejo de las pacientes con márgenes positivos para neoplasia intracervical en la pieza operatoria.

La recomendación en las guías de la Sociedad Americana de Colposcopía y Patología Cervical (ASCCP) sugiere que los pacientes con NIC 1 sean monitorizados y seguidos debido a que la tasa de regresión espontánea es muy alta, por lo que sugiere que el tratamiento excicional sea principalmente dirigido a pacientes con NIC2/3 ${ }^{(11)}$.

El objetivo principal del presente estudio fue determinar la incidencia de recurrencia de NIC en pacientes sometidos a cono LEEP/LLETZ con margen comprometido versus margen negativo.

\section{MATERIALES Y MÉTODOS}

Se realizó un estudio observacional, retrospectivo y analítico en una cohorte de pacientes con diagnóstico de NIC, sometidos al procedimiento de resección con asa electroquirúrgica (LEEP/LLETZ) en el Servicio de Ginecología Oncológica del Instituto Nacional de Enfermedades Neoplásicas (INEN), entre enero del 2002 a diciembre del 2003.

Los criterios de inclusión del estudio fueron: edades entre 18 y 55 años, con diagnóstico de NIC y sometidos a cono LEEP/LLETZ con intención de tratamiento. Los criterios de exclusión fueron: pacientes sometidos a otros tratamientos en el cérvix, como crioterapia, cauterización cervical, conización láser; patología cervical mayor a NIC, presencia de adenocarcinoma in situ, presencia de tumor de la colisión, gestación.

Los participantes del estudio fueron divididos en dos grupos en función de las características del margen de la pieza operatoria, en: 1) margen comprometido o 2) margen negativo. Margen comprometido fue definido como presencia de enfermedad neoplásica intraepitelial en contacto con el borde de sección quirúrgica o hasta los $3 \mathrm{~mm}$ distantes al borde de contacto y margen libre con ausencia de enfermedad neoplásica intraepitelial cervical por encima de los $3 \mathrm{~mm}$ de desde el borde de sección quirúrgica. La presencia de enfermedad recurrencia/ persistencia de NIC la cual, se definió como la presencia de histología de NIC posterior a tratamiento con LEEP seguido de algún control negativo.

El tiempo de recurrencia/persistencia, fue establecido desde la fecha del procedimiento de cono LEEP/LLETZ hasta la presencia histológica de la anormalidad. El método estándar de procedimiento de LEEP/LLETZ se realizó en la sala de cirugía menor bajo anestesia local con anestesia con y sin epinefrina, utilizando para ello Xylocaína $1 \%$ con 1/100,000 unidades de epinefrina inyectados intracervicalmente. Se aplicó ácido acético al 4\%, para determinar la localización de la lesión inicial, posteriormente se aplicó lugol. La resección fue realizada con asa de electrocauterio con posibilidad de realizarla en una pasada o en varias en base a la extensión de la enfermedad. Una vez resecada la lesión, se procedió a electro-fulgurar el lecho expuesto con electrocauterio de bola y posterior colocación de sustancia Monsel y tapón vaginal.

Los procedimientos de cono LEEP/LLETZ fueron realizados en el departamento de Ginecología oncológica del INEN. El 
diagnóstico histológico de la recurrencia/persistencia fue llevado a cabo en el departamento de Patología del INEN, los patólogos no tuvieron acceso la información del tipo de margen en los participantes del estudio.

El seguimiento de los pacientes en forma rutinaria se realizó cada 3 meses con examen físico, PAP y colposcopía si era necesario.

Para el análisis de los datos, las variables numéricas se presentan como promedios y desviación estándar mientras que las variables categóricas se presentan como frecuencias absolutas y relativas.

Las comparaciones entre variables numéricas se llevaron a cabo usando la prueba $T$ de Student y para las variables categóricas con la prueba de Chi cuadrado. Para valorar las diferencias en la recurrencia de NIC entre los participantes con margen comprometido versus margen negativo se calculó la razón de incidencias acumuladas o riesgo relativo (RR) con su respectivo intervalo de confianza (IC) al 95\%.
La significancia estadística se fijo con un valor $\mathrm{p}$ menor a 0.05 . El procesamiento de datos y el análisis estadístico fueron efectuados con programa SPSS versión 12.0.

\section{RESULTADOS}

En total 451 pacientes fueron sometidos a LEEP durante el periodo de estudio. De ellos 342 (75\%) presentaron márgenes negativos posteriores al procedimiento y 109 (25\%) presentaron márgenes comprometidos. Del grupo de pacientes con margen comprometido 94 participantes cumplían con los criterios de elegibilidad y del grupo de pacientes con margen negativo 102 participantes cumplían con los criterios de elegibilidad. Ambos grupos fueron seguidos en busca de recurrencia/persistencia de NIC. De los 94 pacientes con márgenes comprometidos posteriores a cono LEEP, 22 pacientes fueron sometidos a histerectomía complementaria a criterio del médico evaluador y 72 pacientes fueron sometidos a observación. La (Figura 1) resume el flujo de los participantes en el estudio.

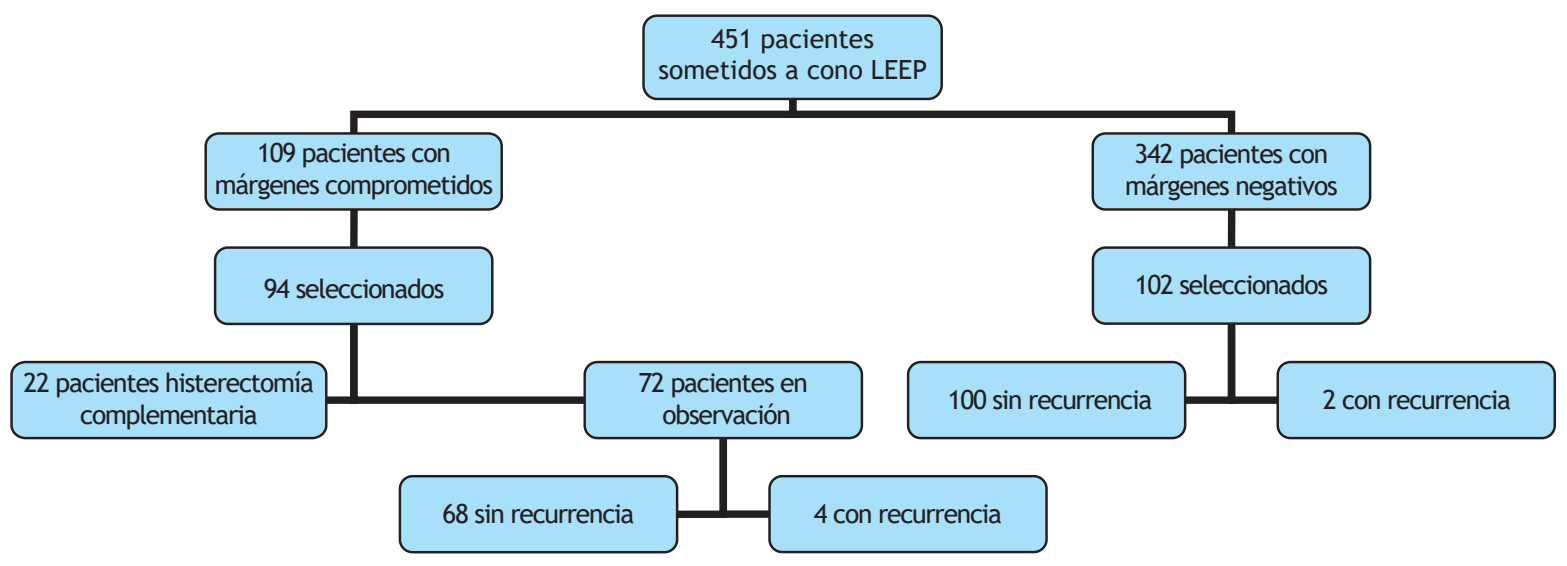

Figura 1. Fluxograma que muestra la distribución de los pacientes sometidos a cono LEEP durante el periodo de estudio

Los pacientes tuvieron una media de seguimiento de 21,74 meses. La edad promedio de los participantes fue 35,32 años (rango 22-52; DE 6,7), el promedio de parejas sexuales (poliandría) encontrada fue de 2,1 parejas (rango 1-6; DE 1) y el promedio del número de hijos de la población estudiada (paridad) fue de 2,7 hijos (rango 0-9; DE 1,9).

Del grupo de pacientes con margen comprometido se encontró NIC 1 , NIC 2 y NIC 3 en 20 (21,27\%), en 32 $(34,04 \%)$, y 42 (44,68\%); respectivamente. De ellos, $29(30,8 \%)$ presentaron compromiso ectocervical y 65 $(69,14 \%)$ compromiso endocervical siendo esta diferencia estadísticamente significativa $(\mathrm{p}<0.01)$.

El promedio de edad para la población de recurrencia/ persistencia fue de 37,2 años (30-43 años) y el tiempo promedio de presentación de la recurrencia/persistencia de 23,93 meses. Al evaluar la presentación de recurrencia/ persistencia en la población con margen comprometido se identificaron 4 casos (5.5\%); de ellos, 3 participantes presentaron compromiso endocervical y 1 participante presentó compromiso ectocervical.

En el grupo con margen negativo, 2 pacientes presentaron recurrencia/persistencia (1,96\%), siendo en ambos casos compromiso ectocervical. No encontramos diferencias estadísticamente significativas entre la incidencia de recurrencia/persistencia de cualquier tipo de NIC al comparar a los participantes con margen comprometido con los de margen negativo, RR= 2.7; IC 95\% (0.41 a 33.8), $\mathrm{p}<0,201$. De los 22 pacientes sometidos a histerectomía complementaria, $8(36,4 \%)$ pacientes presentaron neoplasia residual: $3(37,5 \%)$ casos de NIC 1 y 5 (62,5\%) de NIC 3, siendo el resto de muestras negativas para neoplasia residual $(n=14 ; 63,6 \%)$. La (Tabla 1$)$ evidencia la incidencia de recurrencia/persistencia de NIC según tipo de margen de la lesión. 
Tabla 1. Recurrencia/persistencia de neoplasia intracervical según tipo de margen de la lesión posterior al cono LEEP

\begin{tabular}{|c|c|c|c|}
\hline & $\begin{array}{c}\text { Recurrencia } \\
n(\%)\end{array}$ & $\begin{array}{c}\text { No Recurrencia } \\
n(\%)\end{array}$ & \\
\hline Margen Comprometido & $4(5,5 \%)$ & $90(94,5 \%)$ & $P<0,201$ \\
\hline Margen Negativo & $2(1,96 \%)$ & $100(98,04 \%)$ & \\
\hline
\end{tabular}

\begin{tabular}{|c|c|c|}
\hline \multicolumn{3}{|c|}{ Recurrencia/persistencia vs tipo de NIC } \\
\hline & $\begin{array}{c}\text { Recurrencia/persistencia } \\
\text { Casos }\end{array}$ & $\begin{array}{c}\text { No Recurrencia/persistencia } \\
\text { Casos }\end{array}$ \\
\hline NIC 1 & 1 & 32 \\
\hline NIC 2 & 1 & 39 \\
\hline NIC 3 & 4 & 75 \\
\hline
\end{tabular}

\section{DISCUSIÓN}

El principal hallazgo del estudio fue que no hubo asociación entre el tipo de margen y la presencia de recurrencia/ persistencia de neoplasia intracervical al final de un seguimiento promedio de casi 22 meses.

Este hallazgo es importante dado que la presencia de márgenes negativos en un procedimiento LEEP no elimina probabilidades de recurrencia/persistencia. Con lo cual es importante el seguimiento posterior al LEEP en ambos grupos de pacientes.

En el presente estudio se encontró un porcentaje de recurrencia/persistencia del 5,55\%, estos datos son menores que los reportados por Zhu et al. en el 2015 de $11.3 \%^{(10)}$. El tiempo promedio de presentación de la recurrencia/persistencia fue 21.4 , contrario a los hallazgos encontrados por Skimer et al quien encontró que el 74\% de los pacientes que presentaron recurrencia/persistencia fue dentro de los primeros 6 meses de seguimiento ${ }^{(12)}$.

Como mencionamos, al finalizar el tiempo de seguimiento posterior al uso de LEEP con fines terapéuticos no encontramos diferencias significativas entre el grupo de mujeres con margen comprometido versus las mujeres del grupo con margen negativo en la recurrencia/persistencia de lesiones cervicales pre-malignas. Este hallazgo es similar a lo reportado por Oshea $\mathrm{AO}$ et $\mathrm{al}^{(13)}$, quienes no encontraron asociación entre el estado de los márgenes del cono LEEP, el grado de NIC, o la profundidad de la escisión y la citología anormal posterior; sin embargo, Ramchandani SM et $\mathrm{al}^{(14)}$, reportan que el estado del margen endocervical y la severidad de la neoplasia como factores que predicen la enfermedad permanente o recurrente. Del mismo modo, Malpati $\mathrm{R}^{(14)}$ et al. $\mathrm{y}$ Chikazawa $\mathrm{K}$ et al ${ }^{(16)}$ encontraron similares hallazgos. Esto se debería probablemente al efecto de diatermia sobre la superficie de corte al momento de la remoción de la pieza operatoria, la respuesta inflamatoria asociada y la consecuente activación del sistema inmunitario posterior al procedimiento. El potencial de recurrencia se basa no solo en la remoción completa de la lesión cervical sino también en los hábitos sexuales de las pacientes, su respuesta inmune y la virulencia del $\mathrm{VPH}^{(17)}$. Es así como en el estudio de Anderson et al se determinó que las pacientes con conteo de CD4 < 200 tienen tasas de recurrencia más altas que en valores de CD4 mayor ${ }^{(18)}$. En el presente estudio, los pacientes del grupo de cirugía complementaria presentaron ausencia de enfermedad residual en $63,6 \%$, similar a los datos encontrados por en la revisión hecha por Soto-Wright et al donde se reporta estudios sin enfermedad residual post cirugía complementaria en un rango que va entre el 56 a $92 \%{ }^{(19)}$.

Asimismo, el uso de datos proveniente de registros médico podría ser una limitación del estudio dado que podría generar sesgo de medición debido a la calidad de los datos; no obstante es conocido que el INEN existe un control riguroso del llenado de las historias clínica con lo cual la calidad del dato estaría garantizada.

En conclusión, no se encontró diferencias en la recurrencia/ persistencia de NIC para pacientes con compromiso del margen tras cono LEEP/LLETZ frente a los pacientes con margen libre de enfermedad. Lo que sugeriría según lo encontrado en el presente estudio que los pacientes con resultado de margen comprometido tras cono LEEP/LLETZ podrían ser sometidos a manejo conservador y control periódico, evento que es determinante para esta población ya que las lesiones pre malignas son característicamente identificadas en población potencialmente con intensión de fertilidad y podríamos mejorar así la calidad de vida de las pacientes en su reintegración a la vida normal.

Por lo encontrado en el presente estudio, se sugiere el manejo expectante de los pacientes con compromiso del margen tras cono LEEP/LLETZ, independientemente del tipo de neoplasia intracervical o compromiso endocervical con cuidadoso seguimiento durante los primeros años. Sugiriendo el manejo individualizado del paciente tomando en cuenta la edad, margen, hábitos sexuales, y seguridad de seguimiento. 


\section{REFERENCIAS BIBLIOGRÁFICAS}

1. Anttila A, Kotaniemi-Talonen L, Leinonen M, Hakama $M$, Laurila P, Tarkkanen J, et al. Rate of cervical cancer, severe intraepithelial neoplasia, and adenocarcinoma in situ in primary HPV DNA screening with cytology triage: randomised study within organised screening programme. BMJ. 2010; 340: 1804

2. Reich O, Pickel H, Lahousen M, Tamussino K, Winter R. Cervical intraepithelial neoplasia III: Long-term outcome after cold-knife conization with clear margins. Obstet Gynecol 2001; 97(3):428-30.

3. Duesing N, Schwarz J, Choschzick M, Jaenicke F, Gieseking F, Issa R. Assessment of cervical intraepithelial neoplasia (CIN) with colposcopic biopsy and efficacy of loop electrosurgical excision procedure (LEEP). Arch Gynecol Obstet.2012;286(6):1549-54.

4. Casta S, De Unzo M, Infante F, Bonacita B, Marinelli M, Rubino $A$, et al. Disease persistence in patients with cervical intraepithelial neoplasia undergoing electrosurgical conization. Gynecol oncol. 2002; 85(1):119-24.

5. Serati M, Siesto G, Carollo S, Formenti G, Riva C, Cromi $A$, et al. Risk factors for cervical intraepithelial neoplasia recurrence after conization: a 10-year study. European Journal of Obstetrics \& Gynecology and Reproductive Biology.2012;165:86-90.

6. Fogle RH, Span CO, Ecoley KA, Basil J. Predictors of cervical dyspasia after the loop electrosurgical excision proceduce. J Reprod Med. 2004; 49(6):481-6.

7. Baloglu A., Uysal D, Bezircioglu I, Bicer M, Inci A. Residual and recurrent disease rates following LEEP treatment in highgrade cervical intraepithelial lesions. Arch Gynecol Obstet. 2010; 282(1):69-73.

8. Wu D, Zheng Y, Chen W, Guo C, Yu J, Chen G, et al. Prediction of residual/recurrent disease by HPV Genotype after loop excision procedure for high-grade cervical intraepithelial neoplasia with negative margins. Australian and New Zealand Journal of Obstetrics and Gynaecology. 2011; 51: 114-8.

9. Melli P, Duarte G, Quintana S. Multivariate analysis of risk factors for the persistence of high-grade squamous intraepithelial lesions following loop electrosurgical excision procedure. International Journal of Gynecology and Obstetrics. 2016; 133: 234-7.

10. Zhu M, He Y, Baak JP, Zhou X, Qu Y, Sui L, et al. Factors that influence persistence or recurrence of high-grade squamous intraepithelial lesion with positive margins after the loop electrosurgical excision procedure: a retrospective study. BMC Cancer. 2015;15:744.

11. Massad LS, Einstein MH, Huh WK, Katki H, Kinney W, Schiffman M, et al. 2012 updated consensus guidelines for the management of abnormal cervical cancer screening tests and cancer precursors. J Low Genit Tract Dis. 2013;17:S1-S27.

12. Skimer E, Gehrig P, Van Le L. High grade squamous Intraepithelial Lesions: abbreviating posttreatment surveillance. Obst \& Gynecol. 2004; 103(3): 488-92.

13. Oshea A, Stockdale C. The impact of LEEP margin status on subsequent abnormal cervical cytology. Proceedings in Obstetrics and Gynecology. 2014;4(2):5.

14. Ramchandani SM, Houck KL, Gaughan JP, Roth A. Predicting Persistent/Recurrent Disease in the Cervix After Excisional Biopsy. Medscape General Medicine. 2007;9(2):24.

15. Malapati R, Chaparala S, Cejtin HE. Factors influencing persistence or recurrence of cervical intraepithelial neoplasia after loop electrosurgical excision procedure. J Low Genit Tract Dis. 2011;15(3):177-9.
16. Chikazawa K, Netsu S, Motomatsu S, Konno R. Predictors of recurrent/residual disease after loop electrosurgical excisional procedure. J Obstet Gynaecol Res. 2016; 42(4): 457-63

17. Tillmanns T, Falkner C, Engle D, Wan J, Mannel R, Walker J, et al. Preoperative predictors of positive margins after loop electrosurgical excisional procedure-cone. Gynecol Oncol. 2005;100(2):379-84.

18. Tate D, Anderson R. Recurrence of cervical dysplasia among women who are infected with the human immunodeficiency virus: a case-control analysis. American Journal of Obstetrics and Gynecology.2002; 186(5):880-2.

19. Soto-Wright V, Samuelson R, McLellan R. Current Management of Low-Grade Squamous Intraepithelial Lesion, High-Grade Squamous Epithelial Lesion, and Atypical Glandular Cell. Obst \& Gynecol. 2005; 48(1):147-59.

\section{Fuentes de financiamiento:}

Este artículo ha sido financiado por los autores.

\section{Conflictos de interés:}

Los autores declaran no tener ningún conflicto de interés.

\section{Correspondencia:}

Gino Venegas Rodríguez

Dirección: Av. Brasil 2746, Pueblo Libre.

Liga Peruana de Lucha contra el Cáncer. Lima, Perú.

Teléfono: 998254182

Correo electrónico: gvenegas@ligacancer.org.pe

Recibido: 07 de setiembre de 2016 Aprobado: 05 de febrero de 2017 